\title{
Thymic stromal lymphopoietin-mediated epicutaneous inflammation promotes acute diarrhea and anaphylaxis
}

\author{
Hongwei Han, ${ }^{1}$ Tennille D. Thelen, ${ }^{1,2}$ Michael R. Comeau, ${ }^{3}$ and Steven F. Ziegler ${ }^{1,2}$ \\ IImmunology Program, Benaroya Research Institute, Seattle, Washington, USA. ²Department of Immunology, University of Washington School of Medicine, Seattle, Washington, USA \\ ${ }^{3}$ Inflammation Research, Amgen, Seattle, Washington, USA.
}

\begin{abstract}
Atopic dermatitis (AD) and food allergy are closely linked; however, the mechanisms that guide the progression of $A D$ to allergic inflammatory responses at other mucosal surfaces, including the gastrointestinal tract, are not well understood. Here, we determined that exposure of mice that have been epicutaneously sensitized with thymic stromal lymphopoietin (TSLP) and antigen to repeated oral doses of the same antigen induced acute diarrhea and anaphylaxis. In this model, loss of TSLP signaling specifically in DCs led to loss of induced allergic diarrhea through lack of sensitization. While TSLP responses were not required during oral allergen challenge, $C D 4^{+} \mathrm{T}$ cells were required and transferred disease when introduced into naive hosts. In addition, oral exposure to the antigen prior to skin sensitization blocked development of allergic disease. Finally, mice lacking the receptor for IL-25 failed to develop acute diarrhea and anaphylaxis, highlighting a role for IL-25 in the initiation of type 2 immunity in the intestine. These results demonstrate a role for TSLP and IL-25 in the atopic march from skin sensitization to food allergic responses and provide a model system for the generation of potential therapeutic interventions.
\end{abstract}

\section{Introduction}

Food allergy is a common public health problem, with a prevalence ranging around $8 \%$, and increasing in the last several decades (1-3). Diagnosis of food allergy can be problematic, and currently there is no cure. Thus, individuals with food allergy opt for avoidance of the trigger foods, as early detection is imperfect and medications only manage the symptoms of disease. The immunologic basis of food allergy remains elusive, and new approaches and model systems are needed to identify targets for treatment.

Food allergy is associated with a range of diseases such as asthma, atopic dermatitis (AD), eosinophilic esophagitis (EoE), and exercise-induced anaphylaxis $(1,4,5)$. Additionally, there is an association between skin barrier defects and development of food allergic responses, possibly due to an increased chance of sensitization by allergens permeating the skin, bypassing oral tolerance. Epidemiologic data suggest that sensitization to peanut protein can occur in children through exposure to peanut in oils applied to inflamed skin (6). Also there is a genetic correlation between loss-of-function mutations within the filaggrin gene (Flg) that are associated with $\mathrm{AD}$ and also show an increased risk of peanut allergy $(7,8)$. As food allergy and atopic disease are clearly associated, factors known to be involved with atopic disease might also play a role in food allergy.

Thymic stromal lymphopoietin (TSLP), an IL-7-related cytokine, has emerged as an important regulator of Th2-type responses in the skin, lung, and gastrointestinal (GI) tract $(9,10)$. Recent stud-

Conflict of interest: Michael R. Comeau is an employee of Amgen. Submitted: September 2, 2014; Accepted: October 2, 2014.

Reference information: J Clin Invest. 2014;124(12):5442-5452. doi:10.1172/JCI77798. ies have implicated a role of TSLP in atopic march. For instance, TSLP triggers progression from AD to asthma (11-14). We hypothesize that cutaneous TSLP production also initiates a cascade leading to intestinal inflammation and resulting in the development of food allergy. We have developed a model that demonstrates epicutaneous sensitization in the presence of TSLP leads to Th2-driven allergic responses to that antigen when reexposure occurs in the GI tract. This response requires TSLP-responsive DCs, and is dependent on $\mathrm{CD}^{+} \mathrm{T}$ cells. The signaling through IL-25 and its cognate receptor, IL-17RB, initiates Th2 cytokine responses and allergic disease development following oral allergen challenge.

\section{Results}

Mice overexpressing TSLP in the skin develop GI allergy. Epidemiological studies have shown that cutaneous inflammation associated with $\mathrm{AD}$ is a risk factor for food allergy, demonstrating a link between these 2 conditions (6).We hypothesized that allergen sensitization in the context of AD-like skin inflammation would induce allergic diarrhea following oral challenge with the antigen. To test this hypothesis, we used mice that had a doxycycline-inducible (dox-inducible) TSLP transgene specifically in keratinocytes (K5-rTA TetO-Tslp mice, herein referred to as K5-Tslp; ref. 15). A concentration of dox was used $(0.01 \mathrm{mg} / \mathrm{ml}$ in drinking water) that led to local inflammation but undetectable serum TSLP (data not shown). We sensitized K5-Tslp and normal littermate control mice with OVA (5 times intradermally [i.d.] over a 2.5 -week period) in the presence of dietary dox. After a 3-week rest period, these mice were fed intragastrically (i.g.) with soluble OVA (Figure 1A). Following this protocol, K5-Tslp mice developed canonical features of diarrhea after the third challenge (Figure 1, B and C), including increases in 
A

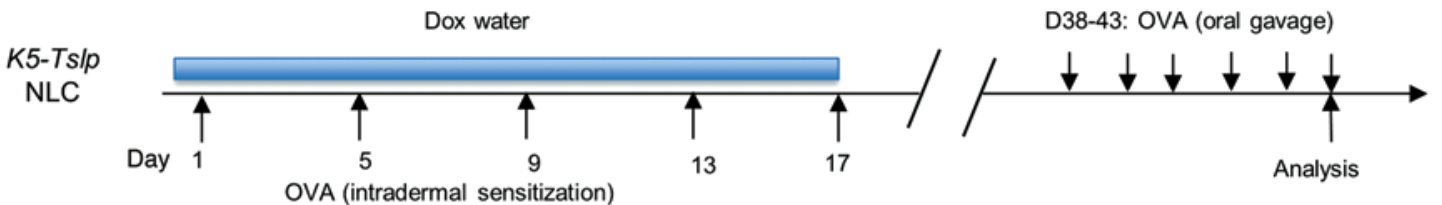

B
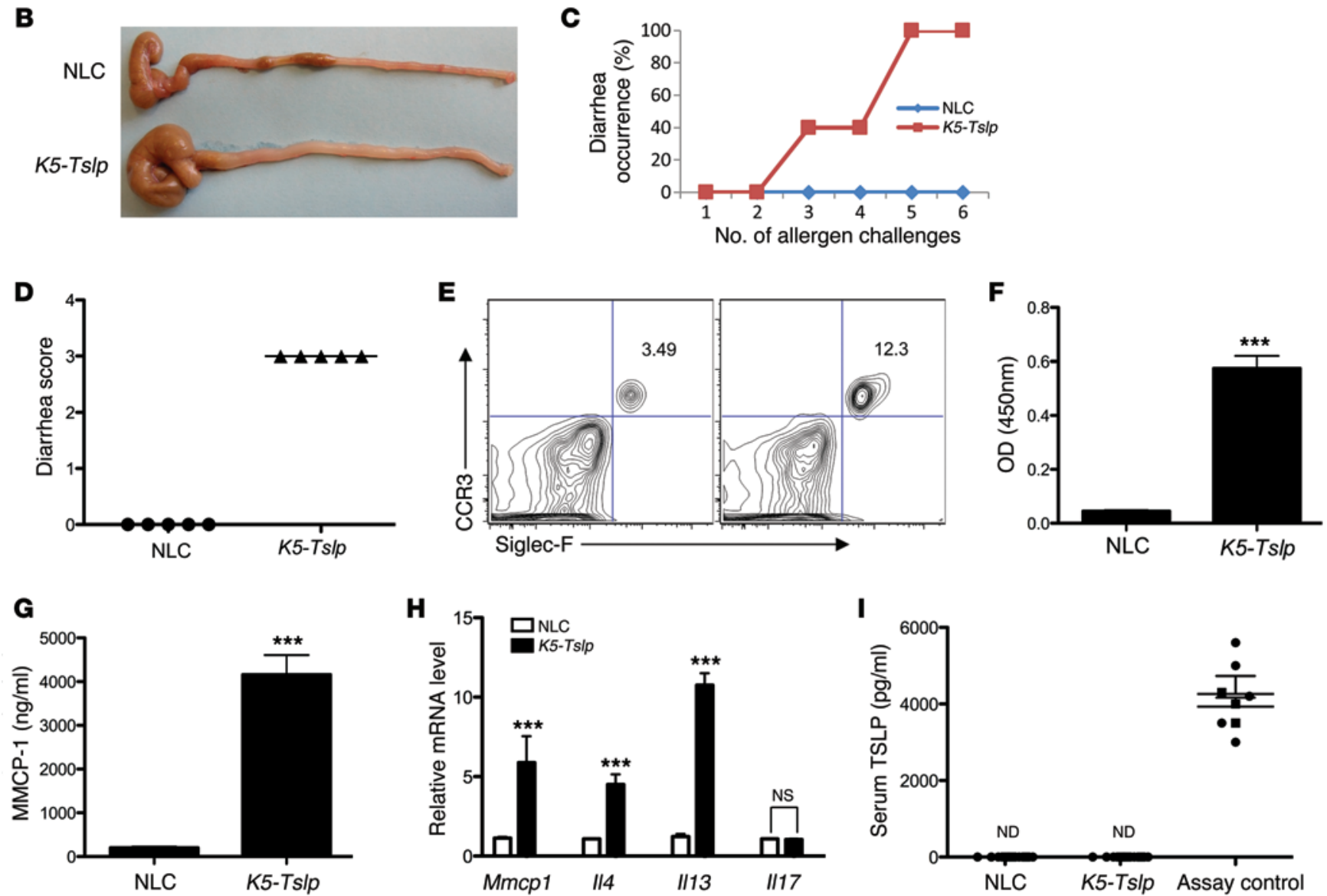

Figure 1. Increased TSLP expression in the skin promotes antigen-induced GI allergy. (A) Experimental protocol. Experimental and control animals were given dietary dox $(0.01 \mathrm{mg} / \mathrm{ml}$ in drinking water) and intradermal OVA as indicated, followed by a rest and oral challenge. (B) Representative photograph of the cecum and colon from indicated mice. NLC, normal littermate control. (C) Diarrhea occurrence. (D) Diarrhea score. (E) Frequencies of peripheral eosinophils. Cells were gated on $\mathrm{CD} 45^{+}$cells, and plots are representative of 10 mice analyzed. (F) OVA-specific serum IgE levels. (C) MMCP-1 serum levels. (H) Mmcp1 and cytokine expression levels in the jejunum. (I) TSLP serum levels. Control K5-Ts/p mice were treated with $1 \mathrm{mg} / \mathrm{ml}$ of dox for 3 weeks and used as assay control. ND, not detected $(<7.8 \mathrm{pg} / \mathrm{ml})$. For $\mathbf{C}, \mathbf{D}$, and $\mathbf{F}-\mathbf{H}$, data are representative of 3 independent experiments with 5 mice per group. Groups of animals were compared using Student's $t$ tests. Error bars indicate the mean \pm SD. ${ }^{* *} P \leq 0.001$.

the frequency of eosinophils and OVA-specific IgE levels and production of mast cell-specific protease MMCP-1 and Th2 cell-associated cytokines IL-4 and IL-13 (Figure 1, D-H). Histopathological analysis of jejunum section showed that K5-Tslp mice had severe eosinophil infiltrates (Supplemental Figure 1; supplemental material available online with this article; doi:10.1172/JCI77798DS1). We did not detect TSLP protein in the circulation (Figure 1I).

Mice sensitized epicutaneously in the presence of TSLP develop GI allergy. We next sought to define the nature of the control mechanisms by developing a mouse model of food allergy in which WT $\mathrm{BALB} / \mathrm{c}$ mice were sensitized with mouse recombinant TSLP and the antigen OVA i.d. to induce an AD-like skin inflammatory disease $(11,16)$. Following a 5 -day rest period, mice were challenged i.g. with OVA for 6 consecutive days (Figure 2A). Mice treated with
TSLP developed acute diarrhea symptoms beginning at the third administration of OVA, and 100\% of mice were symptomatic by the fifth feed (Figure $2 \mathrm{~B}-\mathrm{D}$ ). Diarrhea was also noted by direct observation of the colon and cecum; the liquid stool observed following TSLP+OVA-induced diarrhea contrasts with the solid pellets seen when mouse serum albumin (MSA) was substituted for TSLP during the sensitization phase (Figure 2B). After challenge, the jejunum of TSLP+OVA-sensitized animals had a significant inflammatory cell infiltrate that contained large numbers of eosinophils (Supplemental Figure 2). Consistent with the induction of Th2-type inflammation by TSLP treatment, OVA-specific IgE levels and type 2 cytokine responses were markedly elevated in TSLP+OVA-treated mice (Figure 2, E-G). We also observed an increase in $M m c p 1$ transcript levels (Figure 2, F and $\mathrm{H}$ ) in the jeju- 
A

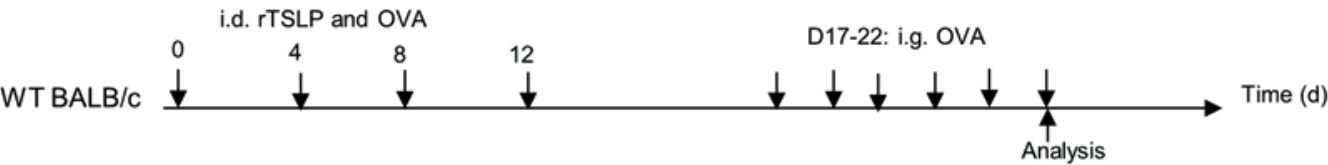

B
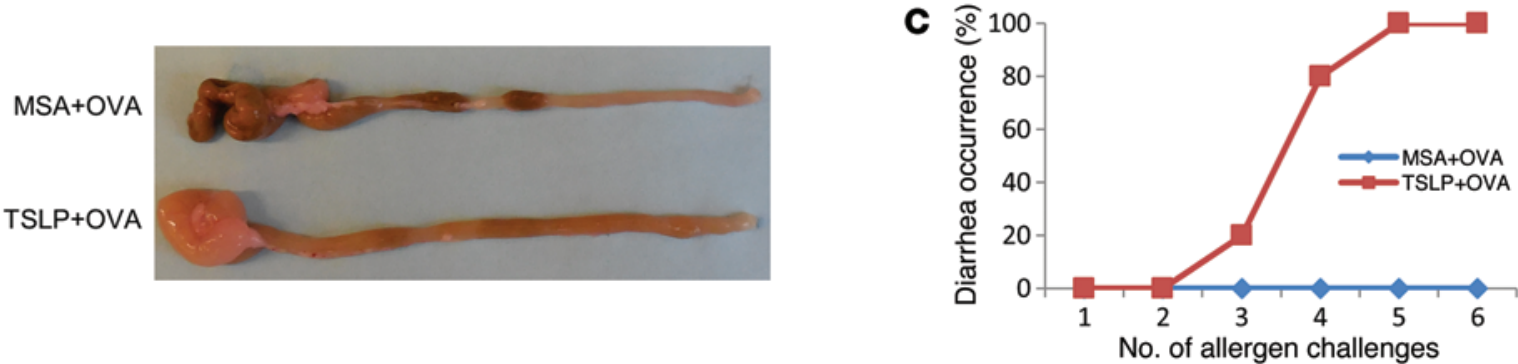
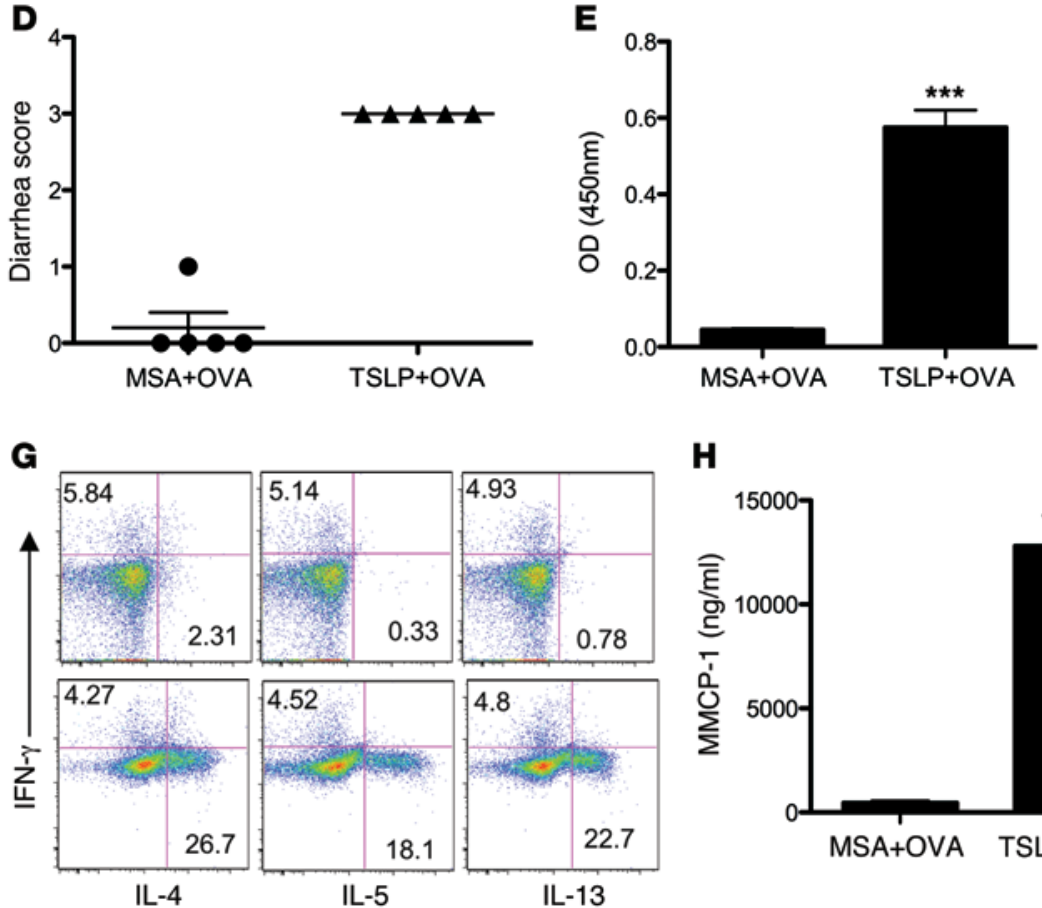

H

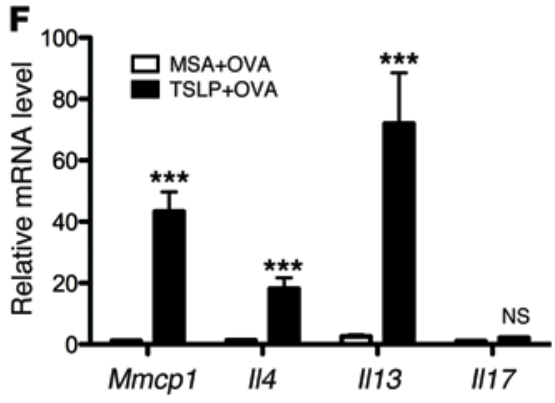

I

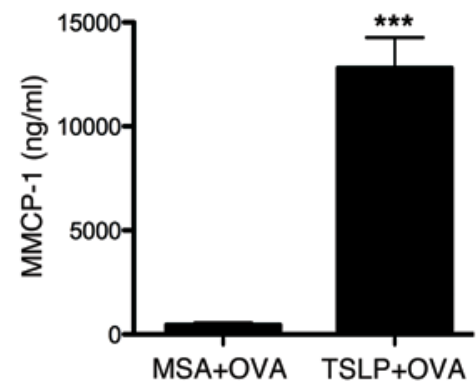

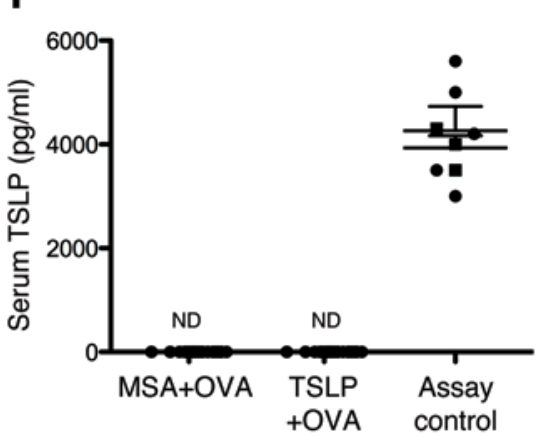

Figure 2. Intradermal administration of TSLP promotes antigen-induced diarrhea. (A) Experimental protocol. (B) Representative cecum and colon from MSA+OVA- and TSLP+OVA-treated mice. (C) Diarrhea occurrence. (D) Diarrhea score. (E) OVA-specific serum IgE levels. (F) Quantitative RT-PCR for Mmcp1 and cytokine gene expression from the jejunum. (G) Intracellular cytokine staining of MLN cells isolated from mice treated with MSA+OVA (upper panels) and TSLP+OVA (lower panels). Plots are gated on CD4+CD44 hi cells and representative of 10 mice analyzed. (H) MMCP-1 serum levels. (I) TSLP serum levels. K5-Ts $/ p$ mice were treated with $1 \mathrm{mg} / \mathrm{ml}$ of dox for 3 weeks and used as assay control. ND, $<7.8 \mathrm{pg} / \mathrm{ml}$. Data are representative of 3 independent experiments with 5 mice per group. Groups of animals were compared using Student's $t$ tests ( $\mathbf{G}$ and $\mathbf{I}$ ). Error bars indicate the mean \pm SD. ${ }^{* * *} P \leq 0.001$.

num, as well as a marked 50-fold increase in MMCP-1 serum levels in TSLP+OVA-sensitized versus MSA+OVA-sensitized control mice, consistent with activated mast cells (Figure $2 \mathrm{H}$ ). TSLP protein in the serum was undetectable $(<7.8 \mathrm{pg} / \mathrm{ml})$, suggesting that circulating TSLP is not required for the development of allergic diarrhea upon rechallenge in this model (Figure 2I).

To determine whether increasing the time between skin exposure to TSLP plus antigen and oral challenge affected development of intestinal inflammation, the interval between skin sensitization and oral challenge was increased from 5 to 21 days. We observed delayed disease onset in this protocol. However, disease severity and Th2 responses were indistinguishable from that shown in Figure 2 for mice rested 5 days (Supplemental Figure 3).
Although OVA is frequently used in studies of $\mathrm{T}$ cell differentiation and allergic inflammation, it is not a clinically relevant allergen. To establish a model with a more clinically relevant food allergen, we repeated the sensitization and challenge using whole peanut extract (WPE). We observed a similar pattern of inflammation in mice that were sensitized and challenged with WPE, suggesting that this can be used as a preclinical model of physiological food allergic responses (Supplemental Figure 4).

TSLP induces oral allergen-induced anaphylaxis. The most common symptoms of food allergy are anaphylactic responses, brought on by the release of preformed chemical mediators by mast cells and basophils, that manifest through the GI tract, skin, and respiratory system. The increase in both local and circulating MMCP-1 
A
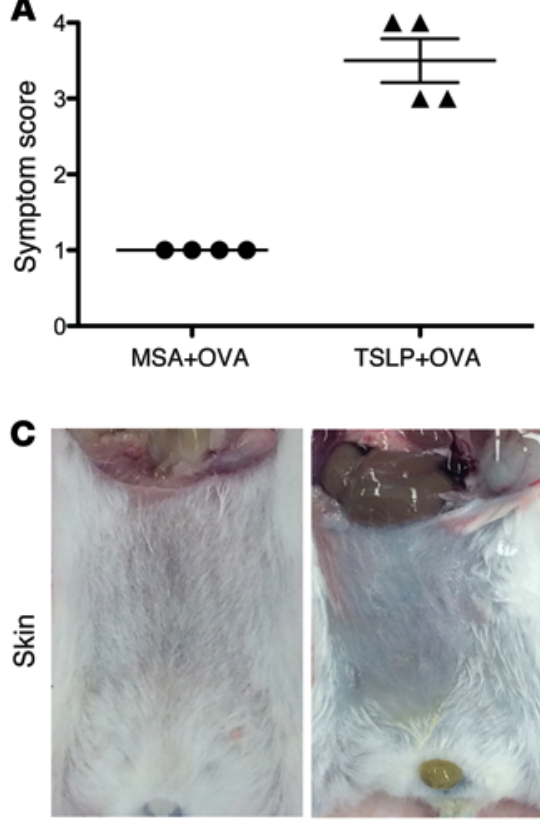

MSA+OVA

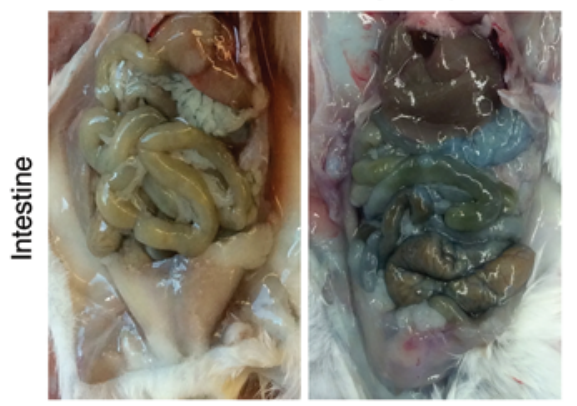

B

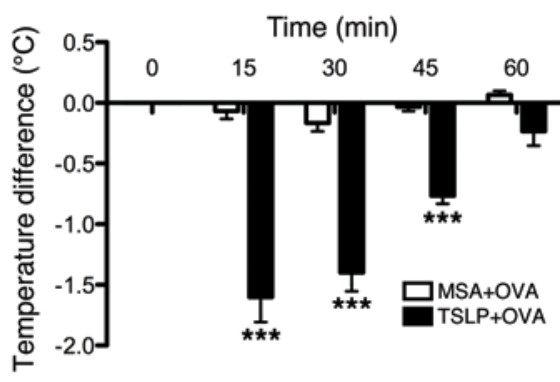

Skin
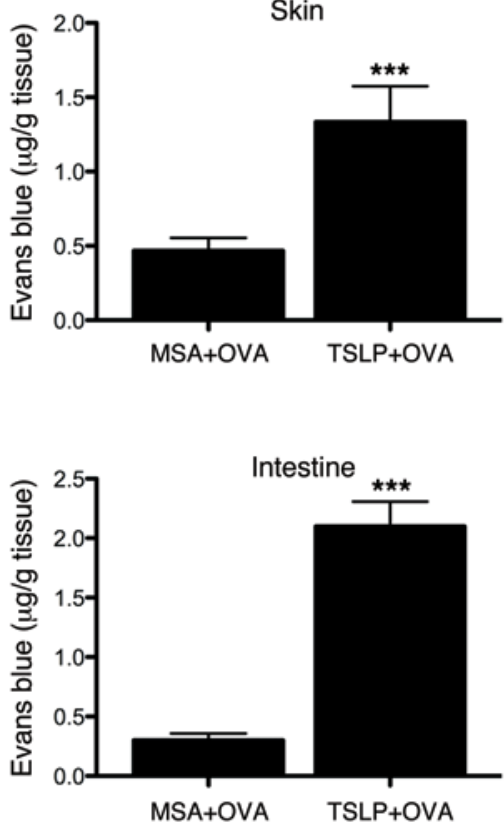

Figure 3. Antigen-driven anaphylaxis in TSLP+OVA-sensitized mice. (A) Symptom scores. (B) Body temperature responses after antigen challenge. (C) Serum leakage at skin and intestine. Data are representative of 3 independent experiments with 4 mice per group. Error bars indicate the mean \pm SD. ${ }^{* * *} P \leq 0.001$

took 2 approaches. First, we sensitized mice with TSLP+OVA as before and then treated them systemically with either an isotype control or a neutralizing TSLP-specific mAb (clone M702) (18) during repeated antigen challenge. As shown in Figure 4, A-D, anti-TSLPtreated mice developed indistinguishable intestinal inflammation following OVA challenge. For the second approach Tslp-deficient mice were sensitized and challenged as above. The Tslp-deficient mice responded to the i.g. challenge as robustly as the Tslp-sufficient mice (Figure 4, E-H), showing that TSLP was not required for the i.g. challenge. We conclude from these studies that increased skin-restricted TSLP levels during the antigen-sensitization phase is sufficient to yield an aggravated response to antigen when subsequently encountered within the intestine.

TSLPR signaling by DCs is required for GI allergy. In order to determine what

was consistent with mast cell activation (Figure $2 \mathrm{~F}$ ). To examine additional aspects of anaphylaxis, we took several approaches, including a scoring system previously used in cholera toxin- and peanut-driven murine models of food-induced anaphylaxis (17). Administration of TSLP+OVA, but not MSA+OVA, resulted in a robust pattern of physiologic symptoms characterized by an increased clinical allergy score after oral challenge with OVA (Figure 3A). Mice that had been sensitized with TSLP also responded to oral OVA challenge with a significant drop in body temperature that was not seen in control mice (Figure 3B).

Individuals with allergic responses to food allergens often present with itchy red rash, which can be modeled in mice by examining vascular permeability following injection with Evans blue dye. We injected mice with Evans blue dye prior to the final oral challenge and monitored serum leakage in the skin and intestine. Mice sensitized with TSLP displayed vascular leakage, as evidenced by increased Evans blue dye in both skin and GI tract (Figure 3C). Taken together, these data demonstrate that anaphylactic and allergic reactions occurred in response to oral OVA challenge after TSLP-driven sensitization.

GI allergy does not require systemic TSLP. The data clearly show that TSLP was required during sensitization phase for the development of food allergy in mice. To begin to determine whether TSLP was also involved directly during the challenge phase, we cell populations require direct responses to TSLP, we took advantage of a mouse strain containing a conditional knockout allele of Tslpr (Tslprfff; Supplemental Figure 5). We first examined DCs, which have been shown to be important for TSLP-mediated type 2 inflammatory responses (19). To test the requirement for TSLPR on DCs to promote allergic immune responses, we crossed Ts $/ \mathrm{pr}^{\mathrm{f} / \mathrm{f} / \mathrm{l}}$ mice with Cd11c-Cre mice (referred to as Cd11c-Cre Tslprf/f) and examined food allergy development using the protocol described above. Control Ts $l p r^{f / f}$ mice developed diarrhea by the fourth challenge. On the other hand, Cd11c-Cre Tslprf/f mice failed to develop allergic diarrhea (Figure 5, A and B). Consistent with the reduction in disease development, Cd11c-Cre Tslprf/f mice had reduced OVAspecific serum IgE levels and Mmcp1, Il4, and Il13 message in the jejunum (Figure 5, C and D). Correspondingly, Cd11c-Cre Tslprf/f $\mathrm{CD}^{+} \mathrm{T}$ cells from the mesenteric lymph nodes (MLNs) produced less IL-4, IL-5, and IL-13, as well as increased IFN- $\gamma$, suggesting defective Th2 cell activation (Figure $5 \mathrm{E}$ ). This shows that TSLPR is required on DCs for control of Th2 intestine responses.

Basophil depletion reduces development of GI allergy. Given the known role of basophils in the context of $\mathrm{AD}$ and $\mathrm{EoE}(20,21)$ as well as the reported role of TSLP in their development and function, we tested whether basophils were required in this model. We treated WT mice with Ba103, a mAb specific for CD200R3 that is an activating receptor expressed by basophils and mast cells (22). 
A

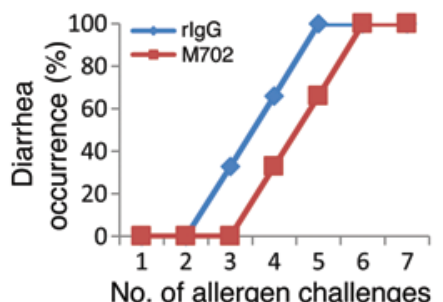

No. of allergen challenges

\section{E}

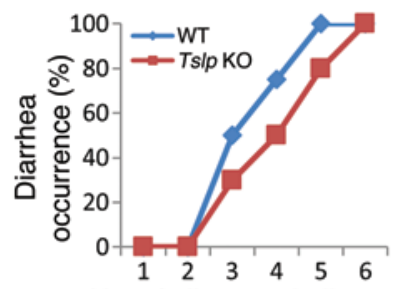

No. of allergen challenges
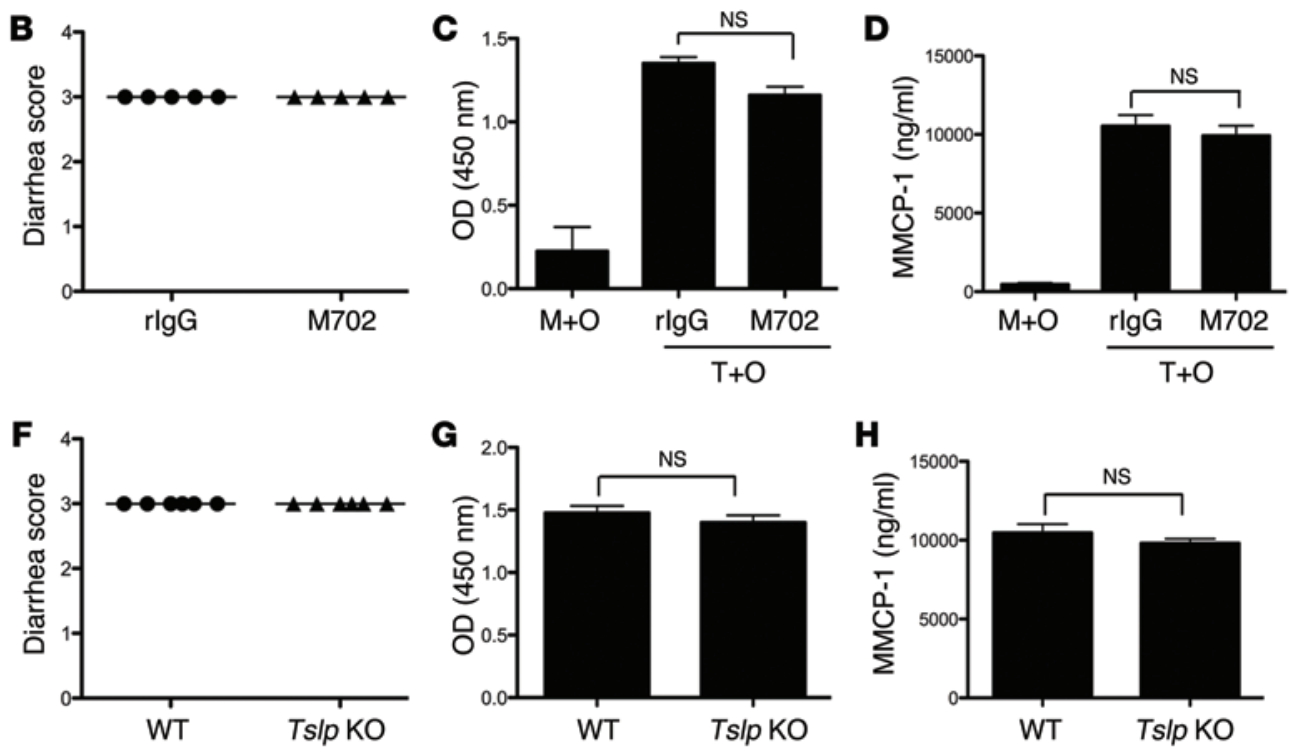

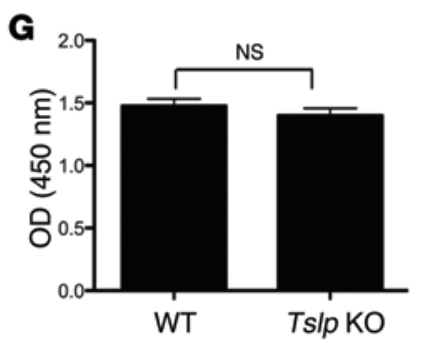

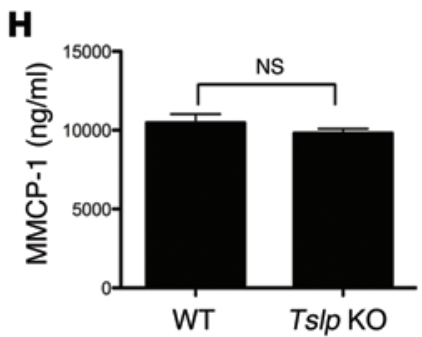

Figure 4. TSLP is dispensable during challenge phase. (A and E) Diarrhea occurrence. $n=10$. (B and $\mathbf{F}$ ) Diarrhea score. (C and $\mathbf{G}$ ) OVA-specific serum IgE levels. ( $\mathbf{D}$ and $\mathbf{H})$ MMCP-1 serum levels. Data are representative of 2 independent experiments with 5 mice per group (B-D and $\mathbf{F}-\mathbf{H})$. M+O, MSA+OVA; $\mathbf{T}+0$, TSLP+OVA. Error bars indicate the mean \pm SD.

Basophil-depleted mice showed attenuated allergic diarrhea and MMCP-1 serum levels (Figure 6, A and B). Furthermore, vascular permeability was significantly reduced in Ba103-treated mice when compared with that of mice receiving the control $\mathrm{Ab}$ (Figure 6C).

As an alternative approach, we used Mcpt8-Dtr transgenic mice (23) in which basophils were transiently depleted by injection of diphtheria toxin (DT), which binds to the human diphtheria toxin receptor (huDTR) on basophils (Supplemental Figure 6A). Although C57BL/6 mice were resistant to developing diarrhea (data not shown), they did show vascular leakage caused by anaphylaxis (Supplemental Figure 6B). Depletion of basophils in Mcpt8-Dtrpos mice protected them from food-induced anaphylaxis, as evidenced by a decrease of Evans blue dye in the intestine (Supplemental Figure 6, B and C). Taken together, these observations implicate the role of basophils in the etiology of food allergy. While it is still possible that basophils do not require direct TSLP signals, they are clearly involved in the response.

TSLP acts on DCs to promote type 2 immunity in the skin. Several cell types, including DCs and basophils, have been shown to respond directly to TSLP in vitro (24-26). To determine whether these cell populations required direct TSLP responsiveness to promote type 2 immune responses in the skin, we performed TSLP+OVA sensitization on Cd11c-Cre Tslprf/f mice as well as WT mice. Upon TSLP+OVA sensitization, control mice displayed increased cellularity in inguinal lymph nodes (ILN), as well as elevated serum OVA-specific IgE (Supplemental Figure 7). In contrast, Cd11c-Cre Tslpr f/f mice showed dramatically reduced cellularity and OVA-specific IgE, resembling sham-treated control animals (Supplemental Figure 7). Thus, we concluded that TSLP directly acts on DCs to promote antigen-specific type 2 responses in the context of an AD-like skin inflammation.

GI allergy is dependent on $C D 4^{+} T$ cells. A Th2-type milieu has been observed in the intestine of subjects with food allergy (27).
To test the role of $\mathrm{CD}^{+} \mathrm{T}$ cells in the pathophysiology seen in this model of food allergy, we used 2 experimental protocols. First, we depleted $\mathrm{CD} 4^{+} \mathrm{T}$ cells by treating mice with anti-CD 4 antibody GK1.5 following sensitization, but prior to i.g. OVA challenge. This led to a nearly complete loss of $\mathrm{CD} 4^{+} \mathrm{T}$ cells. Acute depletion of $\mathrm{CD} 4^{+} \mathrm{T}$ cells also led to absence of disease onset, decreased levels of serum IgE and MMCP-1, and a reduction in expression of genes related to Th2 cytokine responses (Figure 7, $\mathrm{A}-\mathrm{D}$, and data not shown). These data demonstrate that $\mathrm{CD} 4^{+} \mathrm{T}$ cells are required for the induction of intestinal inflammation following skin sensitization.

To more closely examine the nature of the $\mathrm{CD} 4^{+} \mathrm{T}$ cells that were driving food allergy, we isolated and transferred $\mathrm{CD} 4^{+} \mathrm{T}$ cells from skin-draining lymph nodes of sensitized mice into naive mice. Transfer of the primed cells from TSLP+OVA-treated mice resulted in development of allergic diarrhea following oral challenge (Figure 7, E-H) in recipient mice. Disease onset was not observed in mice injected with $\mathrm{CD} 4^{+} \mathrm{T}$ cells from control mice (Figure 7, E-H); therefore, we conclude that $\mathrm{CD} 4^{+} \mathrm{T}$ cells are sufficient to transfer disease in this mouse model of GI food allergy.

Treatment with oral antigen prior to skin sensitization induces tolerance. Previous studies have shown that low-dose oral allergen treatment can induce tolerance, likely through generation of regulatory T cells with suppressor function $(28,29)$. We sought to determine whether oral exposure to OVA was capable of tolerizing mice to subsequent challenge. We provided mice with dietary OVA (in the drinking water) for 5 days, followed by sensitization and challenge as described above. As shown in Figure 8, A-C, mice exposed to oral OVA failed to develop diarrhea and displayed much-reduced clinical allergy score and antigen-specific IgE levels. Th2 responses were reduced to levels seen in control animals and were dramatically decreased (Figure 8D). Tolerance-induced mice were also protected from anaphylaxis demonstrated by reduced serum leakage 


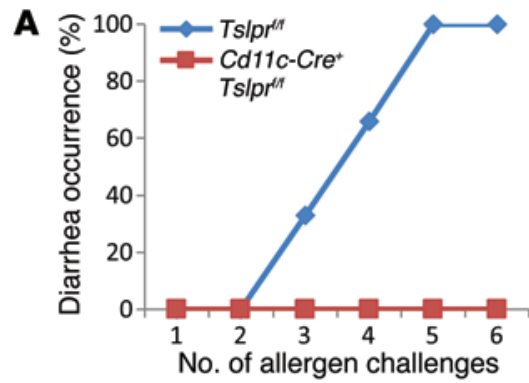

D

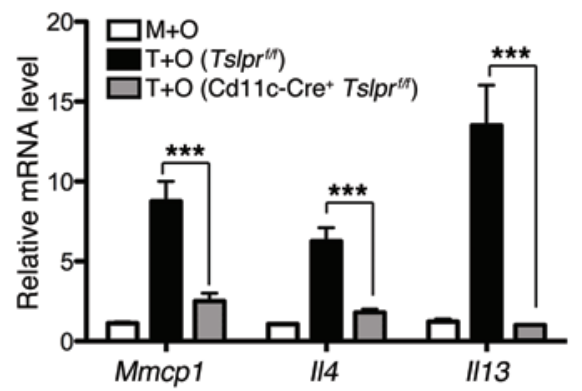

B

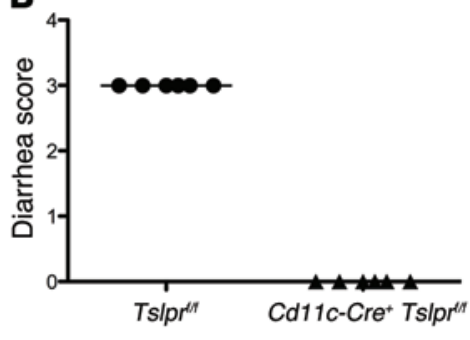

C

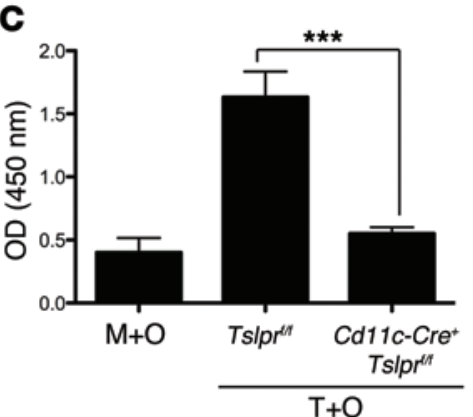

$\mathbf{E}$

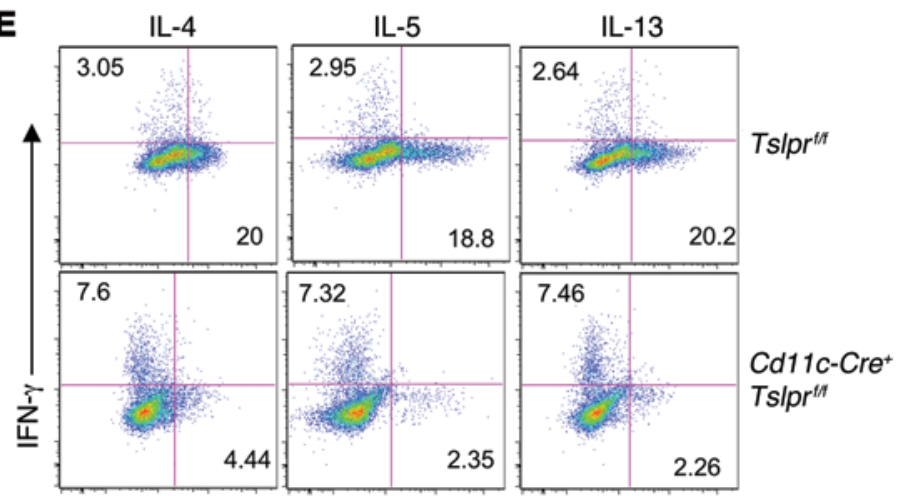

Figure 5. Diminished allergen-induced GI allergy in Cd11c-Cre TsIprf/f mice. (A) Diarrhea occurrence. $n=6$. (B) Diarrhea score. $n=6$. (C) OVA-specific serum IgE levels. (D) Mmcp1 and type 2 cytokine expression levels in the jejunum. (E) Intracellular cytokine staining of MLN cells isolated from a Ts/pr/ff mouse and a Cd11c-Cre Ts/pr/ff mouse. Plots are gated on CD4+CD44 hi cells and representative of 6 mice analyzed. For $\mathbf{C}$ and $\mathbf{D}$, data are representative of 3 independent experiments with 3 mice per group. Error bars indicate the mean $\pm \mathrm{SD}$. ${ }^{* *} P \leq 0.001$.

(Figure $8 \mathrm{G}$ ). These data show that naive mice can be tolerized to antigen before epicutaneous sensitization.

Mice lacking IL-25 signaling fail to develop GI allergy. The data presented above showed that TSLP signaling by DCs was critical for sensitization, but was not involved in the challenge phase. Thus, we focused on other factors and observed increased Il25 expression in the jejunum of mice sensitized with TSLP+OVA and challenged with oral OVA (Figure 9A). We next sought to determine in vivo contributions of IL-25 to GI allergy by taking advantage of mice genetically deficient in the IL-25 receptor $\left(\mathrm{Il} 17 \mathrm{rb}^{-/-}\right.$; ref. 30) using the model described above (Figure 2A). Il17rb-deficient mice displayed a lack of acute diarrhea, reduced Th2 responses, and a decrease of Evans blue dye in the intestine, demonstrating IL-25 as an important effector molecular in the pathogenesis of food allergy (Figure 9, B-E).

\section{Discussion}

Epidemiological data have suggested that children with $\mathrm{AD}$ are also at an increased risk of allergic diarrhea $(1,4,5)$. Recent evidence demonstrating elevated expression of the cytokine TSLP in the lesional skin of $\mathrm{AD}$ patients (31) as well as the finding that TSLP-mediated epicutaneous sensitization can lead to aggravated airway inflammatory responses (11-14) suggested a mechanism for the development of food-allergic responses following epicutaneous sensitization. To test this hypothesis and to dissect the mechanisms leading to allergic diarrhea, we developed a murine model in which skin sensitization to a model food allergen in the presence of TSLP followed by oral challenge with the same antigen resulted in acute allergic diarrhea. We found that TSLP-responsive DCs were required for development of diarrhea and that the onset of allergic diarrhea following oral challenge was TSLP independent. For the challenge phase of the response, we found that CD4 ${ }^{+}$ T cells were both necessary and sufficient to drive diarrhea. These data support a model whereby TSLP present in the skin promotes sensitization to food allergens through differentiation of Th2 cells and in which acute allergic diarrhea was driven by memory CD $4^{+}$ $\mathrm{T}$ cells responding to challenge with the same antigen.

Recent work has suggested that DCs and basophils cooperate to promote Th2 differentiation following appropriate sensitization $(20,32,33)$. Studies in humans and mice have shown that TSLP conditions DCs to induce Th2 cell differentiation (34). In addition, TSLP has been shown to be required for the recruitment of basophils into lymph nodes at the initiation of immune responses (24, 35). Interestingly, we have found that TSLP-responsive DCs are required for the development of allergic diarrhea and that there is a lack of Th2 priming in mice with Tslpr specifically deleted in DCs. Depletion of basophils reduces development of GI allergy, indicating that basophils are involved in the response.

The relative roles of DCs and basophils in the initiation and progression of type 2 inflammatory responses is not clear. For example, it was recently shown that basophils can act as antigenpresenting cells (APCs) in some models of type 2 inflammation (35-37). However, type 2 immunity after house dust mite (HDM) inhalation requires DCs, but not basophils. In this study, basophils did not take up antigen and present it to T cells, whereas cells in a subset of inflammatory DCs that express FceRI are the primary 
A

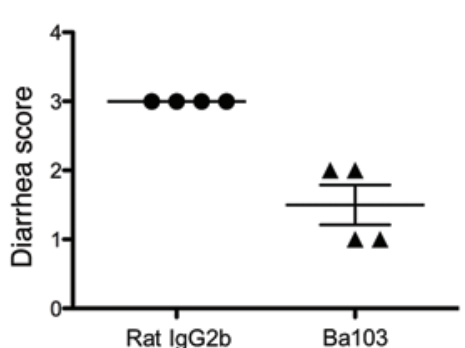

B

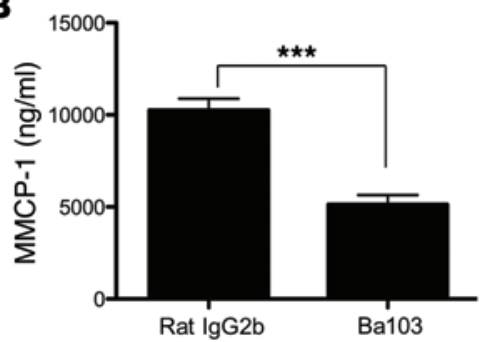

C

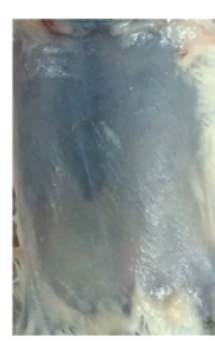

Rat lgG2b

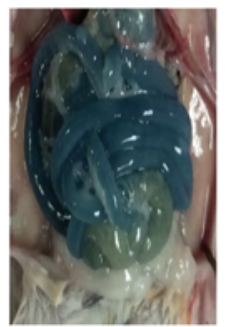

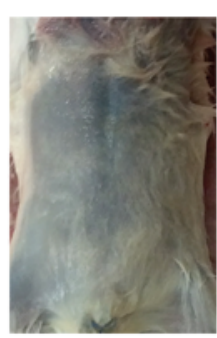

Ba103

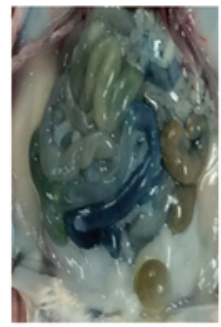

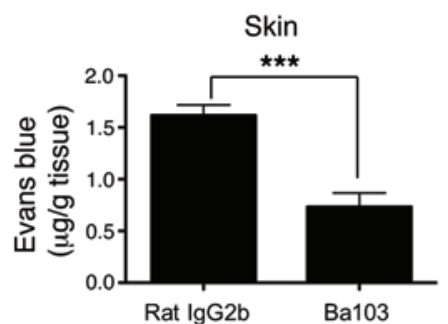

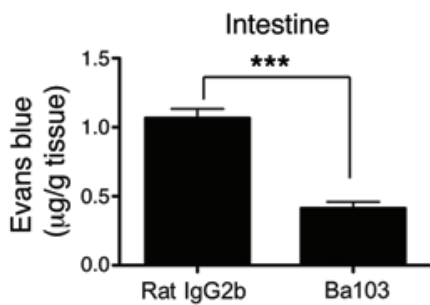

Figure 6. Basophil depletion attenuates GI allergy. (A) Diarrhea score. (B) MMCP-1 serum levels. (C) Serum leakage at skin and intestine. Data are representative of 2 independent experiments with 4 mice per group. Error bars indicate the mean \pm SD. ${ }^{* *} P \leq 0.001$.

population that takes up antigen and drives Th2 differentiation (38). In addition, Otsuka et al. found that basophils were able to drive Th2 differentiation following epicutaneous sensitization with hapten or peptide antigens, while priming with protein antigens required DCs (39). Thus, it appears that the relative roles of these cell types in the initiation of type 2 inflammatory responses is context dependent and may reflect the nature of the antigen and the strength of the accompanying inflammation.

During the preparation of this manuscript, the important roles of basophils and TSLP in a cutaneously sensitized food allergy model were reported $(40,41)$. However, the models used in those studies differed from the current model in that high systemic levels of TSLP were present. In our model, TSLP expression is restricted to the skin and is not found in the circulation. While circulating TSLP has been detected in infants with severe $\operatorname{AD}(42,43)$, levels in children with food allergy have not been reported, with the exception of eosinophilic esophagitis (44-46). Finally, whether TSLP-induced inflammation is mediated by direct TSLP responses by basophils needs to be investigated. Previous studies did not rule out the possibility that TSLP probably triggers other cells to release factors to attract or act on basophils. This possibility is under investigation using mice lacking Tslpr specifically on basophils.
A
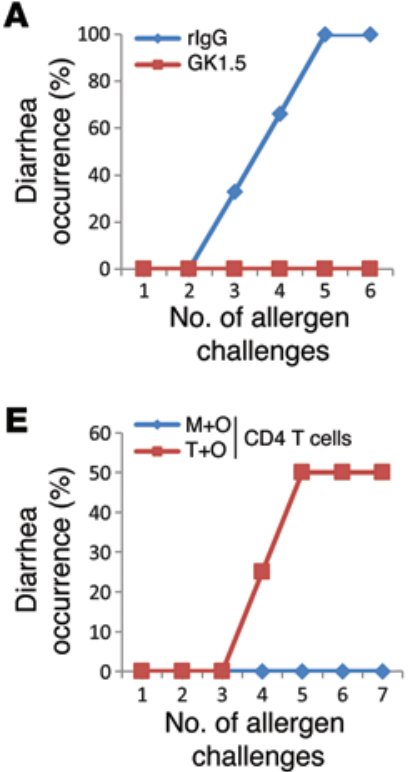

B

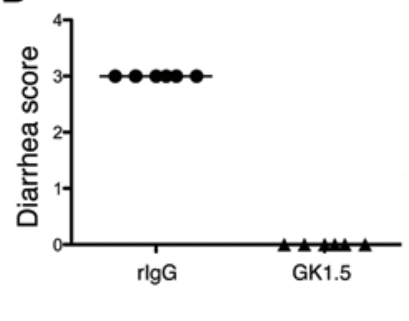

$\mathbf{F}$

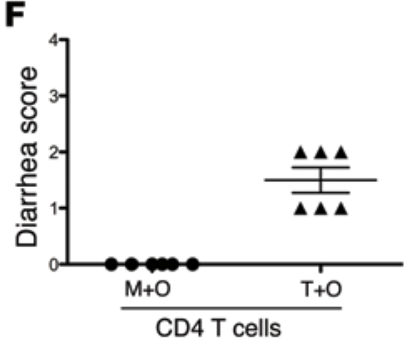

C
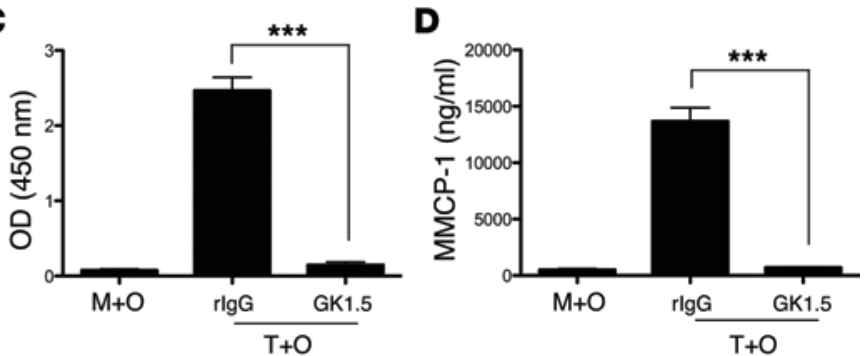

G

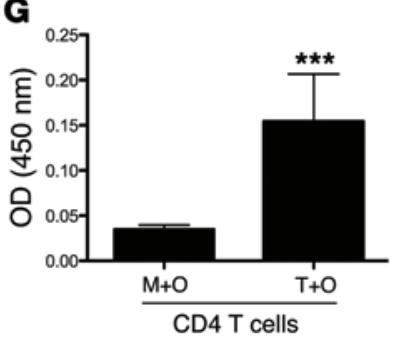

H

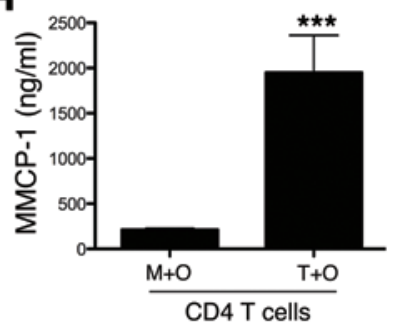

Figure 7. CD4 ${ }^{+} \mathbf{T}$ cells are required for TSLP-mediated GI allergy. (A and E) Diarrhea occurrence. (B and F) Diarrhea score. (C and G) OVA-specific serum IgE levels. ( $\mathbf{D}$ and $\mathbf{H}$ ) MMCP-1 serum levels. Data are representative of 2 independent experiments with 4 to 6 mice per group. Groups of animals were compared using Student's $t$ tests $(\mathbf{G}$ and $\mathbf{H})$. Error bars indicate the mean $\pm S D$. ${ }^{* *} P \leq 0.001$. 
A

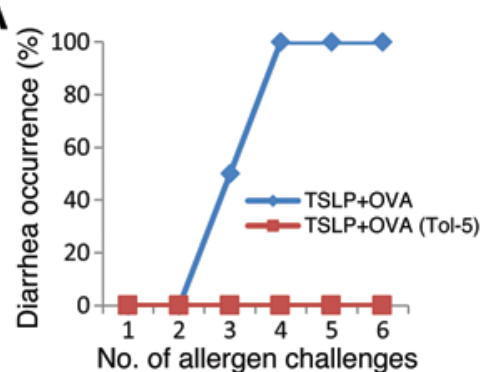

B

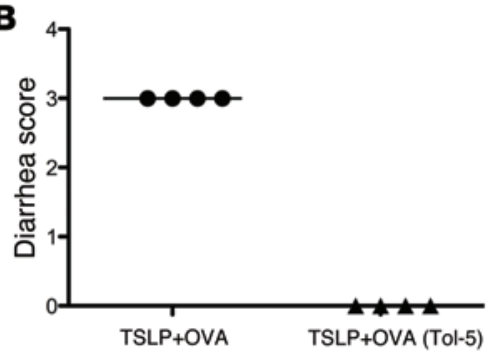

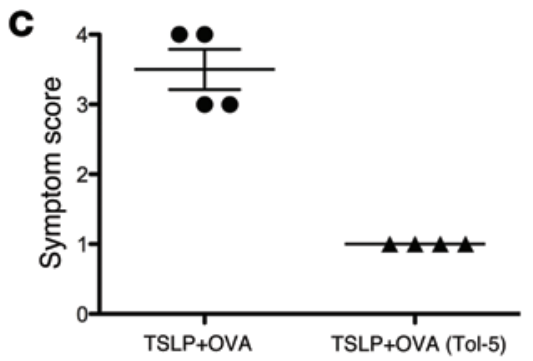

D
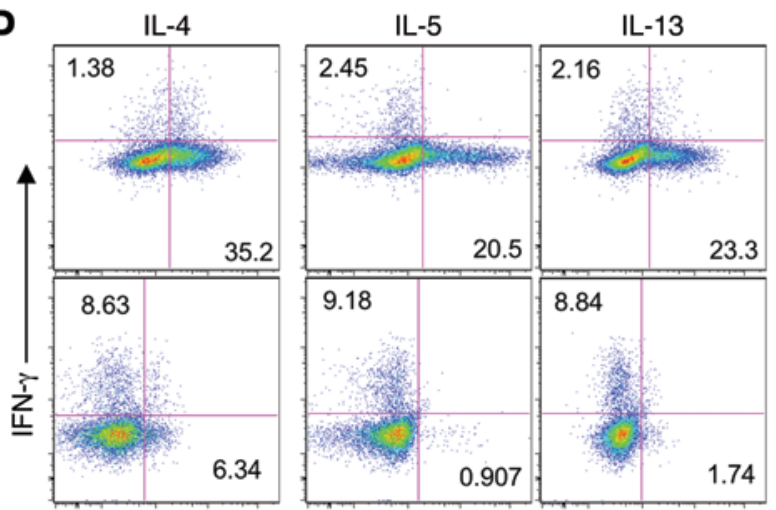

E

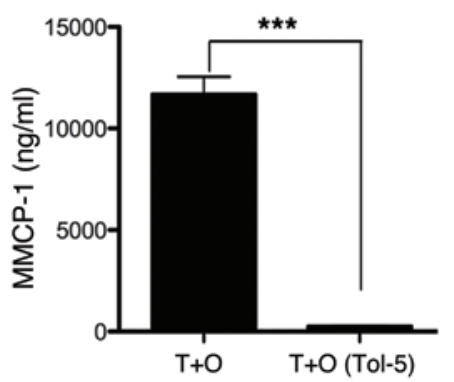

$\mathbf{F}$

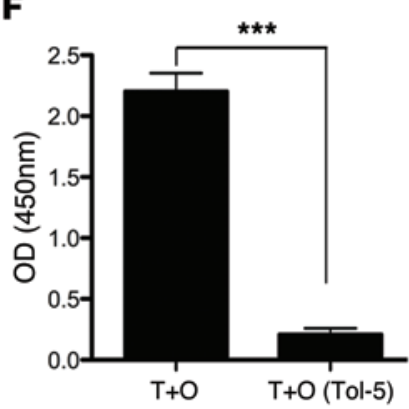

G

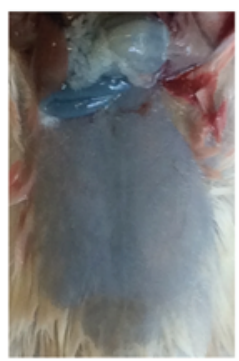

TSLP+OVA

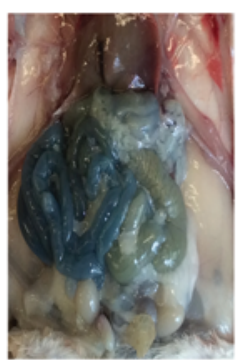

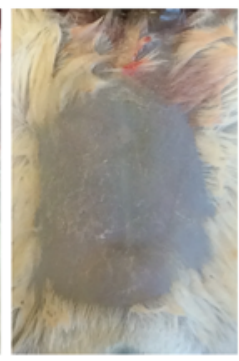

TSLP+OVA (Tol-5)

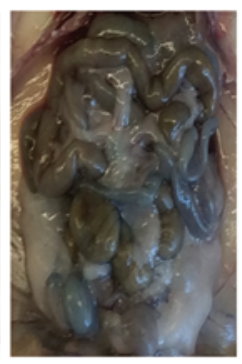

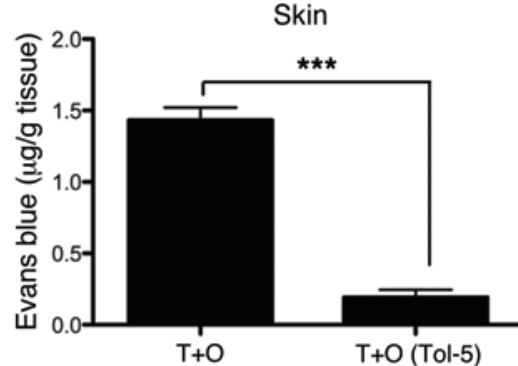

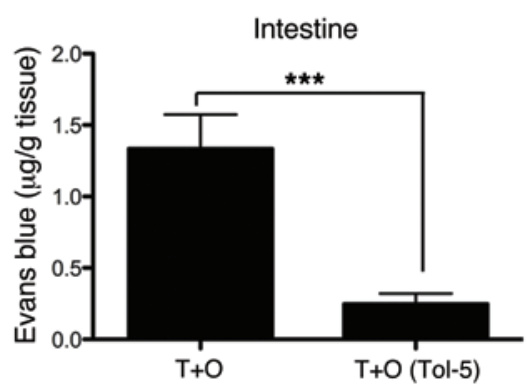

Figure 8. Antigen feeding before skin sensitization suppresses $\mathrm{Gl}$ allergy. (A) Diarrhea occurrence. (B) Diarrhea score. (C) Symptom scores. (D) Intracellular cytokine staining of MLN cells isolated from mice fed with normal water (upper panels) and $1 \%$ OVA in drinking water (lower panels). Plots are gated on $\mathrm{CD} 4^{+} \mathrm{CD} 44^{\text {hi }}$ cells. (E) MMCP-1 serum levels. (F) OVA-specific serum IgE levels. (G) Serum leakage at skin (upper panels) and intestine (lower panels). Data are representative of 2 independent experiments with 4 mice per group. Error bars indicate the mean $\pm \mathrm{SD}$. ${ }^{* * *} P \leq 0.001$.
Since Tslp-deficient mice developed indistinguishable allergic diarrhea and blockade of TSLP did not change Th2 cytokinemediated response during challenge phase (Figure 4), one would expect other factor(s) having an impact on the ultimate outcome of the pathologic responses in this model. Given the fact that the IL-25 message was induced in the jejunum of challenged mice (Figure 9A), together with the data showing Il17rb-deficient mice were protected from food allergy (Figure 9, B-E), the IL-25 pathway could be a major target for postsensitized individuals to prevent food allergy. Consistent with our data on the role of IL-25 in this model, Aalberse et al. have found elevated circulating levels of IL-25 in peanut-allergic children following allergen challenge (47).
Taken together, these data suggest an interplay between epithelial cell-derived molecules and cells that produce Th2 cytokines that may subsequently mediate allergic inflammation at mucosal sites.

Taken together, our findings that identified a role of TSLP and DCs in allergic diarrhea, coupled with our previous finding that TSLP is involved in progression from AD to asthma, support the hypothesis that skin may be an important site for systemic sensitization to certain allergens and shed light on the relationship between $\mathrm{AD}$ and food allergy. The finding that TSLP functions during epicutaneous sensitization to drive the differentiation of allergen-specific Th2 cells but is dispensable during subsequent oral challenge suggests that other factors are involved during this phase of the response. 


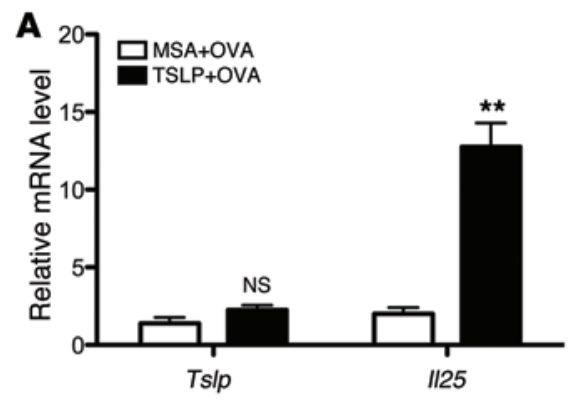

D

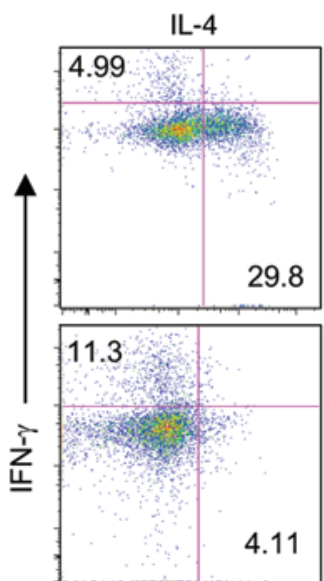

B

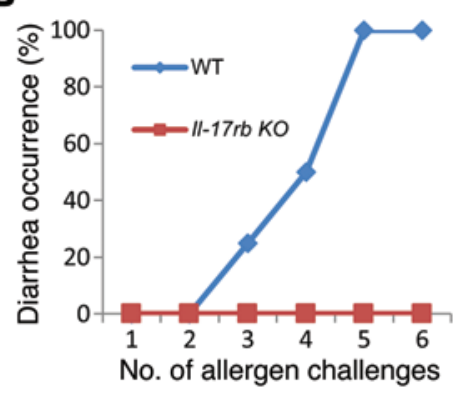

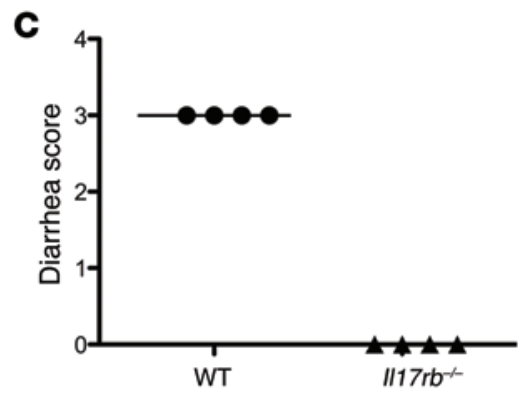

E
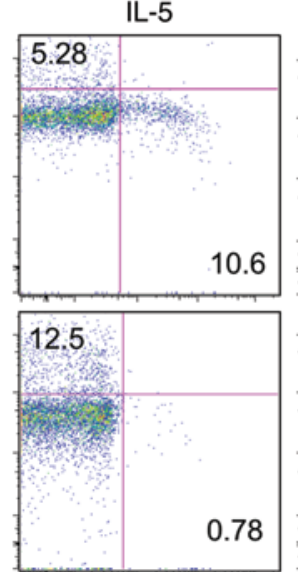

IL-13
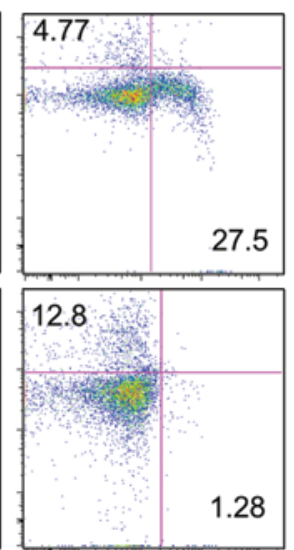

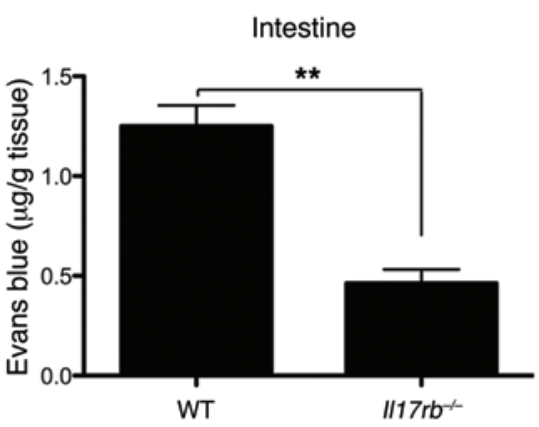

Figure 9. IL-25 signaling is required to induce GI allergy. (A) Quantitative RT-PCR for Ts/p and II25 gene expression from the jejunum following oral challenge. (B) Diarrhea occurrence. (C) Diarrhea score. (D) Intracellular cytokine staining of MLN cells isolated from WT mice (upper panels) and IL17rb-deficient mice (lower panels). Plots are gated on $\mathrm{CD}^{+} \mathrm{CD} 44^{\mathrm{hi}}$ cells and representative of 4 mice analyzed. (E) Serum leakage at intestine. Data are representative of 3 independent experiments with 3 to 4 mice per group. Error bars indicate the mean \pm SD. ${ }^{* *} P \leq 0.01$.

\section{Methods}

Mice and treatments. Six- to eight-week-old female BALB/c mice were obtained from Charles River Laboratories. K5-Tslp and Tslp-deficient mice were generated as described previously (15). Mcpt8-Dtr mice were provided by Adrian Piliponsky (Seattle Children's Hospital, Seattle, Washington, USA). Il17rb-deficient mice were provided by Andrew McKenzie (Medical Research Council Laboratory of Molecular Biology, Cambridge, United Kingdom). The Tslpr-targeting construct (Supplemental Figure 5) was electroporated into embryonic stem (ES) cells, and neomycin-resistant clones were screened by Southern blot analysis. Two independently derived ES clones were injected into blastocysts. Chimeric male offspring were mated to FLP deleter mice. Transmission of the targeted allele was determined by PCR from genomic DNA. Conditional Tslpr-deficient mice were moved to a BALB/c background using marker-assisted accelerated backcrossing technology at DartMouse. Bacterial artificial chromosome transgenic Cd11c-Cre mice were backcrossed 10 generations onto a BALB/c background and then crossed to floxed Tslpr mice on a BALB/c background. All animals were housed in specific pathogen-free conditions in the Benaroya Research Institute animal facility. Intradermal injections were performed as previously described (11, 16). Briefly, $5 \mu \mathrm{g}$ TSLP (Amgen) or MSA (Sigma-Aldrich) with $10 \mu \mathrm{g}$ OVA (A7642; Sigma-Aldrich) was injected i.d. in a $100 \mu \mathrm{l}$ volume of sterile PBS 4 times during a 12-day period. The i.g. challenges with $50 \mathrm{mg}$ of OVA (grade V, A5503; Sigma-Aldrich) were performed as described previously (48). WPE was prepared from unsalted uncooked peanuts by using $20 \mathrm{mmol} / \mathrm{l}$ Tris buffer, as previously described (49).
Total protein concentration of WPE was determined with bicinchoninic acid (BCA) (Pierce). Mice demonstrating profuse liquid stool were recorded as diarrhea-positive animals. A detailed clinical score was assessed 20 to 40 minutes after final oral feeding ( 0 , normal; 1, soft; 2, running; 3, liquid; 4, bloody). Symptom scoring was performed after the fourth challenge according to previously described parameters of symptoms in murine food allergy (17). For depletion with TSLP-specific mAbs, mice were injected with $500 \mu \mathrm{g}$ of control IgG or TSLP-specific mAbs (clone M702; Amgen) i.p. on days 15 and 19.

Vascular permeability measurements. Vascular permeability was evaluated by measuring the leakage of Evans blue dye into the skin and intestine as described before (41).

Measurement of body temperature. The changes in body temperature of mice were measured using a Dual Laser IR Thermometer (42509; Extech), at 0, 15, 30, 45, and 60 minutes after the fifth OVA challenge.

Oral antigen administration. Oral tolerance to OVA was induced by ad libitum administration of 1\% OVA (A5378; Sigma-Aldrich) solution dissolved in drinking water for 5 consecutive days.

Cell culture. MLNs were isolated and cultured in RPMI medium with $10 \%$ fetal calf serum, penicillin, and streptomycin and with 100 $\mu \mathrm{g} / \mathrm{ml}$ OVA for 72 hours. Cells were stimulated with PMA and ionomycin in the presence of brefeldin A for 4 to 5 hours. The cells were harvested and analyzed for cytokine production; further analysis was performed using FlowJo software (Tree Star Inc.).

Depletion of $C D 4^{+} T$ cells and adoptive transfers. Animals were i.p. injected on days 14 and 19 with $200 \mu \mathrm{g}$ of anti-CD4 antibody (clone: 
GK1.5, UCSF Monoclonal Antibody Core) or $200 \mu \mathrm{g}$ rIgG (SigmaAldrich) in a total volume of $200 \mu \mathrm{l}$ PBS. Adoptive transfers were performed as described before $(11,16)$.

Basophil depletion. For basophil depletion by DT, Mcpt8-Dtr mice were treated with $500 \mathrm{ng}$ of DT administered i.p. every 4 to 5 days. For depletion with the Ba103 mAb, WT mice were i.p. injected with $50 \mu \mathrm{g}$ of Ba103 (gift of Hajime Karasuyama, Tokyo Medical and Dental University, Tokyo, Japan) or control rat Ig2b antibodies every 6 days starting from day -2 .

Histology, ELISA, and real-time PCR. Jejunum tissue was collected 10 to $12 \mathrm{~cm}$ distal to the stomach. For histological analysis, tissues were fixed in $10 \%$ formalin and stained with H\&E. MMCP-1 levels were measured by ELISA according to the manufacturer's instruction (eBioscience). Real-time PCR analysis was performed as described before $(11,16)$. Samples were normalized to GAPDH and displayed as fold induction over controls.

Statistics. Statistical analyses were performed with Prism software (GraphPad). Two means were compared using the nonparametric 2-tailed Mann-Whitney $t$ test. Three or more means were compared using 1-way ANOVA with Tukey's post-hoc test. A $P$ value of less that 0.05 was considered significant.

Study approval. All mice were certified to be specific pathogenfree and cared for in accordance with the guidelines of the Institutional Animal Care and Use Committee at Benaroya Research Institute. All animal studies were approved by the IACUC at the Benaroya Research Institute.

\section{Acknowledgments}

We thank the members of the Ziegler laboratory for discussion. We thank Sylvia McCarty for administrative assistance in the preparation of this manuscript. This work was partially supported by NIH grants AI068731, HL098067, AR059058, and AR056113 (to S.F. Ziegler).

Address correspondence to: Steven F. Ziegler, Benaroya Research Institute, 1201 9th Avenue, Seattle, Washington 98101, USA. Phone: 206.344.7950; E-mail: sziegler@benaroyaresearch.org.
1. Burks AW, et al. ICON: food allergy. J Allergy Clin Immunol. 2012;129(4):906-920.

2. Allen KJ, Dharmage SC. The role of food allergy in the atopic march. Clin Exp Allergy. 2010;40(12):1439-1441.

3. Soller L, et al. Overall prevalence of self-reported food allergy in Canada. J Allergy Clin Immunol. 2012;130(4):986-988.

4. Sicherer SH, Leung DY. Advances in allergic skin disease, anaphylaxis, and hypersensitivity reactions to foods, drugs, and insects in 2012. J Allergy Clin Immunol. 2013;131(1):55-66.

5. Rance F. Food allergy in children suffering from atopic eczema. Pediatr Allergy Immunol. 2008;19(3):279-284.

6. Lack G, Fox D, Northstone K, Golding J. Factors associated with the development of peanut allergy in childhood. N Engl JMed. 2003;348(11):977-985.

7. Brown SJ, et al. Loss-of-function variants in the filaggrin gene are a significant risk factor for peanut allergy. J Allergy Clin Immunol. 2011;127(3):661-667.

8. Tan HT, et al. Filaggrin loss-of-function mutations do not predict food allergy over and above the risk of food sensitization among infants. JAllergy Clin Immunol. 2012;130(5):1211-1213.

9. Ziegler SF. Thymic stromal lymphopoietin and allergic disease. JAllergy Clin Immunol. 2012;130(4):845-852.

10. Ziegler SF, Artis D. Sensing the outside world: TSLP regulates barrier immunity. Nat Immunol. 2010;11(4):289-293.

11. Han H, et al. Thymic stromal lymphopoietin (TSLP)-mediated dermal inflammation aggravates experimental asthma. Mucosal Immunol. 2012;5(3):342-351.

12. Leyva-Castillo JM, Hener P, Jiang H, Li M. TSLP produced by keratinocytes promotes allergen sensitization through skin and thereby triggers atopic march in mice. J Invest Dermatol. 2013;133(1):154-163.

13. Demehri S, et al. Notch-deficient skin induces a lethal systemic B-lymphoproliferative disorder by secreting TSLP, a sentinel for epidermal integ- rity. PLoS Biol. 2008;6(5):e123.

14. Zhang Z, et al. Thymic stromal lymphopoietin overproduced by keratinocytes in mouse skin aggravates experimental asthma. Proc Natl Acad Sci U S A. 2009;106(5):1536-1541.

15. Yoo J, et al. Spontaneous atopic dermatitis in mice expressing an inducible thymic stromal lymphopoietin transgene specifically in the skin. J Exp Med. 2005;202(4):541-549.

16. Jessup HK, et al. Intradermal administration of thymic stromal lymphopoietin induces a $\mathrm{T}$ cell- and eosinophil-dependent systemic Th2 inflammatory response. JImmunol. 2008;181(6):4311-4319.

17. Ganeshan K, Neilsen CV, Hadsaitong A, Schleimer RP, Luo X, Bryce PJ. Impairing oral tolerance promotes allergy and anaphylaxis: a new murine food allergy model. J Allergy Clin Immunol. 2009;123(1):231-238.

18. Larson RP, et al. Dibutyl phthalate-induced thymic stromal lymphopoietin is required for th2 contact hypersensitivity responses. Jimmunol. 2010;184(6):2974-2984.

19. Pulendran B, Artis D. New paradigms in type 2 immunity. Science. 2012;337(6093):431-435.

20. Siracusa MC, Kim BS, Spergel JM, Artis D. Basophils and allergic inflammation. J Allergy Clin Immunol. 2013;132(4):789-801.

21. Noti M, et al. Thymic stromal lymphopoietinelicited basophil responses promote eosinophilic esophagitis. Nat Med. 2013;19(8):1005-1013.

22. Kojima T, et al. Mast cells and basophils are selectively activated in vitro and in vivo through CD200R3 in an IgE-independent manner. JImmunol. 2007;179(10):7093-7100.

23. Wada T, et al. Selective ablation of basophils in mice reveals their nonredundant role in acquired immunity against ticks. JClin Invest. 2010;120(8):2867-2875.

24. Siracusa MC, et al. TSLP promotes interleukin-3independent basophil haematopoiesis and type 2 inflammation. Nature. 2011;477(7363):229-233.

25. Zhou B, et al. Thymic stromal lymphopoietin as a key initiator of allergic airway inflammation in mice. Nat Immunol. 2005;6(10):1047-1053.
26. Omori M, Ziegler S. Induction of IL-4 expression in CD4(+) T cells by thymic stromal lymphopoietin. JImmunol. 2007;178(3):1396-1404.

27. Knight AK, Blazquez AB, Zhang S, Mayer L, Sampson HA, Berin MC. CD4 T cells activated in the mesenteric lymph node mediate gastrointestinal food allergy in mice. Am J Physiol Gastrointest Liver Physiol. 2007;293(6):G1234-G1243.

28. Mucida D, Kutchukhidze N, Erazo A, Russo M, Lafaille JJ, Curotto de Lafaille MA. Oral tolerance in the absence of naturally occurring Tregs. J Clin Invest. 2005;115(7):1923-1933.

29. Faria AM, Weiner HL. Oral tolerance: therapeutic implications for autoimmune diseases. Clin Dev Immunol. 2006;13(2):143-157.

30. Neill DR, et al. Nuocytes represent a new innate effector leukocyte that mediates type-2 immunity. Nature. 2010;464(7293):1367-1370.

31. Soumelis V, et al. Human epithelial cells trigger dendritic cell mediated allergic inflammation by producing TSLP. Nat Immunol. 2002;3(7):673-680.

32. Tang $\mathrm{H}$, et al. The $\mathrm{T}$ helper type 2 response to cysteine proteases requires dendritic cell-basophil cooperation via ROS-mediated signaling. Nat Immunol. 2010;11(7):608-617.

33. Leyva-Castillo JM, et al. Skin thymic stromal lymphopoietin initiates Th2 responses through an orchestrated immune cascade. Nat Commun. 2013;4:2847.

34. Liu YJ, et al. TSLP: an epithelial cell cytokine that regulates $\mathrm{T}$ cell differentiation by conditioning dendritic cell maturation. Annu Rev Immunol. 2007;25:193-219.

35. Perrigoue JG, et al. MHC class II-dependent basophil-CD $4^{+} \mathrm{T}$ cell interactions promote $\mathrm{T}(\mathrm{H}) 2$ cytokine-dependent immunity. Nat Immunol. 2009;10(7):697-705.

36. Sokol CL, Chu NQ, Yu S, Nish SA, Laufer TM, Medzhitov R. Basophils function as antigen-presenting cells for an allergen-induced T helper type 2 response. Nat Immunol. 2009;10(7):713-720.

37. Yoshimoto T, et al. Basophils contribute to T(H)2IgE responses in vivo via IL-4 production and presentation of peptide-MHC class II complexes to 
CD4 ${ }^{+}$T cells. Nat Immunol. 2009;10(7):706-712.

38. Hammad $\mathrm{H}$, et al. Inflammatory dendritic cells-not basophils-are necessary and sufficient for induction of Th2 immunity to inhaled house dust mite allergen. J Exp Med. 2010;207(10):2097-2111.

39. Otsuka A, et al. Basophils are required for the induction of Th2 immunity to haptens and peptide antigens. Nat Commun. 2013;4:1739.

40. Noti M, et al. Exposure to food allergens through inflamed skin promotes intestinal food allergy through the thymic stromal lymphopoietin-basophil axis. J Allergy Clin Immunol. 2014;133(5):1390-1399.

41. Muto T, et al. The role of basophils and pro- allergic cytokines, TSLP and IL-33, in cutaneously-sensitized food allergy. Int Immunol. 2014;26(10):539-549.

42. Lee EB, Kim KW, Hong JY, Jee HM, Sohn MH, Kim KE. Increased serum thymic stromal lymphopoietin in children with atopic dermatitis. Pediatr Allergy Immunol. 2010;2(2):e457-e460.

43. Yao W, et al. Interleukin-9 is required for allergic airway inflammation mediated by the cytokine TSLP. Immunity. 2013;38(2):360-372.

44. Noti M, et al. Thymic stromal lymphopoietinelicited basophil responses promote eosinophilic esophagitis. Nat Med. 2013;19(8):1005-1013.

45. Rothenberg ME, et al. Common variants at $5 \mathrm{q} 22$ associate with pediatric eosinophilic esophagitis.
Nat Genet. 2010;42(4):289-291.

46. Sherrill JD, et al. Variants of thymic stromal lymphopoietin and its receptor associate with eosinophilic esophagitis. J Allergy Clin Immunol. 2010;126(1):160-165.

47. Aalberse JA, van Thuijl AO, Meijer Y. Plasma IL-25 is elevated in a subgroup of patients with clinical reactivity to peanut. Clin Transl Allergy. 2013;3(1):40-43.

48. Brandt EB, et al. Mast cells are required for experimental oral allergen-induced diarrhea. JClin Invest. 2003;112(11):1666-1677.

49. Koppelman SJ, et al. Peanut allergen Ara h 3: isolation from peanuts and biochemical characterization. Allergy. 2003;58(11):1144-1151. 